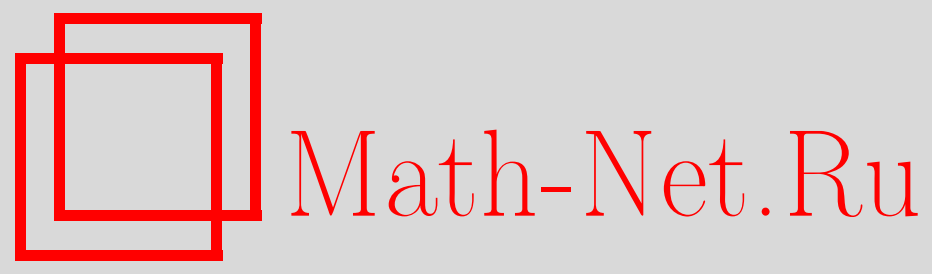

В. А. Емеличев, В. Н. Кричко, Об устойчивости паретовского оптимума векторной задачи булева программирования, Дискрет. матем., 1999, том 11, выпуск 4, 27-32

DOI: https://doi.org/10.4213/dm395

Использование Общероссийского математического портала Math-Net.Ru подразумевает, что вы прочитали и согласны с пользовательским соглашением http://www . mathnet.ru/rus/agreement

Параметры загрузки:

IP : 54.224 .187 .69

26 апреля 2023 г., 13:31:53

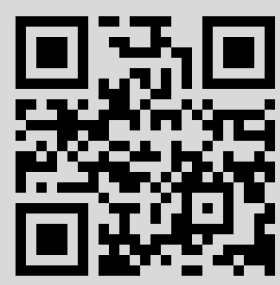




\section{Об устойчивости паретовского оптимума векторной задачи булева программирования}

(C) 1999 г. В. А. Емеличев, В. Н. Кричко

Указаны границы изменений входных параметров векторной задачи линейного булева программирования, сохраняющие парето-оптимальность решения.

Работа выполнена при поддержке Белорусского Республиканского фонда фундаментальных исследований, проект $Ф$ 97-266.

\section{1. Введение}

В работе [1] указан радиус устойчивости единственного оптимального решения классической (скалярной) задачи линейного булева программирования в случае, когда возмущению подвергаются одновременно и коэффициенты линейного функционала и элементы матрицы ограничений. В настоящей заметке с помощью аналогичной техники доказательств выводится формула радиуса устойчивости паретовского оптимума векторной задачи булева программирования. В качестве следствия получено выражение для радиуса квазиустойчивости этой задачи.

\section{2. Определения и вспомогательные результаты}

Будем в основном придерживаться обозначений, а по возможности и терминологии работы [1].

Пусть

$$
C=\left\{c_{i j}\right\} \in \mathbf{R}^{k \times n}, \quad A=\left\{a_{i j}\right\} \in \mathbf{R}^{m \times n}, \quad b \in \mathbf{R}^{m}, \quad k \geqslant 1, \quad n \geqslant 2, \quad m \geqslant 1,
$$

$E^{n}$ - множество вершин единичного $n$-мерного куба.

Рассмотрим $k$-критериальную (векторную) задачу булева программирования

$$
\begin{gathered}
C x \rightarrow \max , \\
A_{i} x \leqslant b_{i}, \quad i \in N_{m}, \quad x \in E^{n} .
\end{gathered}
$$

Здесь $N_{m}=\{1,2, \ldots, m\}, A_{i}=\left(a_{i 1}, a_{i 2}, \ldots, a_{i n}\right)$ и в дальнейшем нижний индекс $i$ у матрицы (вектора) будет указывать на $i$-ю строку (компоненту) соответствующей матрицы (соответствующего вектора). 
Условимся для двух векторов $u, v \in \mathbf{R}^{k}$ считать, что

$$
\begin{aligned}
& u \leqslant v \Longleftrightarrow u_{i} \leqslant v_{i}, \quad i \in N_{k}, \\
& u<v \Longleftrightarrow u_{i}<v_{i}, \quad i \in N_{k} .
\end{aligned}
$$

Будем говорить, что вектор $u$ доминирует вектор $v$, если $u \geqslant v$ и $u \neq v$.

Пусть $X$ - множество всех точек, удовлетворяющих системе (2). Под паретовским оптимумом (или эффективным решением) задачи (1)-(2), как обычно, будем понимать элемент множества Парето

$$
P=\{x \in X: p(x)=\varnothing\}
$$

где

$$
p(x)=\left\{x^{\prime} \in X: C x^{\prime} \geqslant C x, C x^{\prime} \neq C x\right\} .
$$

Другими словами точка $x^{0} \in X$ является паретовским оптимумом задачи (1)-(2) тогда и только тогда, когда для всякой точки $x \in X$ вектор $C x$ не доминирует вектор $C x^{0}$.

Под устойчивостью паретовского оптимума $x^{0}$ будем понимать свойство сохранения эффективности этого оптимума при малых возмущениях всех параметров задачи (1)-(2).

В дальнейшем под $x^{0}$ будем понимать некоторый паретовский оптимум задачи (1)-(2).

Возмущенной задачей назовем следующую векторную задачу:

$$
\begin{gathered}
\left(C+C^{\prime}\right) x \rightarrow \max , \\
\left(A_{i}+A_{i}^{\prime}\right) x \leqslant b_{i}+b_{i}^{\prime}, \quad i \in N_{m}, \quad x \in E^{n} .
\end{gathered}
$$

Расстояние между задачами (1)-(2) и (3)-(4) определим числом $\max \left\{\left\|C^{\prime}\right\|,\left\|A^{\prime}\right\|,\left\|b^{\prime}\right\|\right\}$, где $A^{\prime}-$ матрица со строками $A_{i}^{\prime}, i \in N_{m}, b^{\prime}=\left(b_{1}^{\prime}, b_{2}^{\prime}, \ldots, b_{m}^{\prime}\right)$, $\|\cdot\|$ - чебышевская норма $l_{\infty}$ в соответствующем пространстве. Через $\|\cdot\|^{*}$ будем, как обычно, обозначать норму $l_{1}$ в сопряженном пространстве.

Определение 1. Радиусом устойчивости точки $x^{0} \in X$, являющейся паретовским оптимумом задачи (1)-(2), называется инфимум расстояния от этой задачи до задач (3)-(4), для которых точка $x^{0}$ не является паретовским оптимумом.

Этот радиус будем обозначать через $\rho_{0}\left(x^{0}, A, b, C\right)$ или просто $\rho_{0}$. Расстоянием между системами (2) и (4) назовем число $\max \left\{\left\|A^{\prime}\right\|,\left\|b^{\prime}\right\|\right\}$.

Определение 2. Точка $x \in X$ называется $\varepsilon$-устойчивым решением системы (2), если $x$ - решение любой системы (4), находящейся от (2) на расстоянии, не превосходящем $\varepsilon$.

Дублируя [1], введем обозначения

$$
\begin{gathered}
\gamma_{i}(x)=\frac{A_{i} x-b_{i}}{\|x\|^{*}+1}, \quad i \in N_{m}, \\
\beta(x)=\max _{i \in N_{m}} \gamma_{i}(x), \quad \nu(x)=-\beta(x) .
\end{gathered}
$$

Нам понадобятся некоторые леммы, доказанные в [1]. 


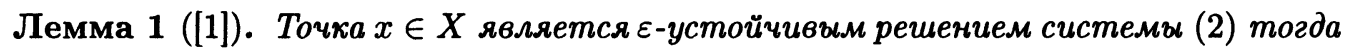
и только тогда, когда выполняется неравенство $\varepsilon \leqslant \nu(x)$.

Пусть $x \in E^{n} \backslash X$. Обозначим через $\beta^{\prime}(x)$ расстояние от системы (2) до ближайшей из систем (4), имеющих точку $x$ в качестве решения.

Лемма 2 ([1]). Для всякой точки $x \in E^{n} \backslash X$ справедливо равенство $\beta(x)=\beta^{\prime}(x)$.

Определение 3. Точку $x^{0}$ назовем $\varepsilon$-устойчивой относительно точки $x$, если для любой матрицы $C^{\prime}$ такой, что $\left\|C^{\prime}\right\|<\varepsilon$, вектор $\left(C+C^{\prime}\right) x$ не доминирует вектор $\left(C+C^{\prime}\right) x^{0}$.

Введем функцию

$$
\alpha\left(x^{0}, x\right)=\max _{i \in N_{k}} \frac{C_{i}\left(x^{0}-x\right)}{\left\|x-x^{0}\right\|^{*}}, \quad x \in E^{n} \backslash x^{0} .
$$

Лемма 3. Пусть $\varepsilon>0$. Точка $x^{0}$ является $\varepsilon$-устойчивой относительно точки $x$, если $\alpha\left(x^{0}, x\right)>\varepsilon$.

Доказательство. Пусть индекс $j$ таков, что

$$
\alpha\left(x^{0}, x\right)=\frac{C_{j}\left(x^{0}-x\right)}{\left\|x-x^{0}\right\|^{*}} .
$$

Тогда при $\left\|C^{\prime}\right\|<\varepsilon$

$$
\begin{aligned}
\left(C_{j}+C_{j}^{\prime}\right)\left(x-x^{0}\right) & <C_{j}\left(x-x^{0}\right)+\varepsilon\left\|x-x^{0}\right\|^{*} \\
& <C_{j}\left(x-x^{0}\right)+\alpha\left(x^{0}, x\right)\left\|x-x^{0}\right\|^{*}=0 .
\end{aligned}
$$

Отсюда получаем, что вектор $\left(C+C^{\prime}\right) x$ не доминирует вектор $\left(C+C^{\prime}\right) x^{0}$. Следовательно, согласно определению $3 x^{0}-\varepsilon$-устойчивая точка относительно точки $x$.

Лемма 3 доказана.

Лемма 4. Если точка $x^{0} \varepsilon$-устойчива относительно точки $x$, то $\alpha\left(x^{0}, x\right) \geqslant \varepsilon$.

Доказательство. Доказательство проведем методом от противного. Пусть точка $x^{0}$ $\varepsilon$-устойчива относительно $x$ и пусть $\varepsilon>\beta>\alpha \geqslant 0$, где $\alpha=\alpha\left(x^{0}, x\right)$.

Рассмотрим возмущающую матрицу $C^{\prime}=\left\{c_{i j}^{\prime}\right\} \in \mathbf{R}^{k \times n}$, элементы которой для любых индексов $i \in N_{k}$ и $j \in N_{n}$ определяются по формуле

$$
c_{i j}^{\prime}=\beta \operatorname{sign}\left(x-x^{0}\right)_{j}
$$

Так как $\left\|C^{\prime}\right\|=\beta<\varepsilon$, в силу $\varepsilon$-устойчивости точки $x^{0}$ относительно точки $x$ вектор $\left(C+C^{\prime}\right) x$ не доминирует вектор $\left(C+C^{\prime}\right) x^{0}$.

В то же время, полагая $e=(1, \ldots, 1)^{T} \in E^{k}$, находим, что

$$
\begin{aligned}
\left(C+C^{\prime}\right)\left(x-x^{0}\right) & =C\left(x-x^{0}\right)+\beta e\left\|x-x^{0}\right\|^{*}>C\left(x-x^{0}\right)+\alpha e\left\|x-x^{0}\right\|^{*} \\
& =C\left(x-x^{0}\right)+e \max \left\{C_{i}\left(x^{0}-x\right), i \in N_{k}\right\} \\
& =C\left(x-x^{0}\right)-e \min \left\{C_{i}\left(x-x^{0}\right), i \in N_{k}\right\} \geqslant 0 .
\end{aligned}
$$

Следовательно, вектор $\left(C+C^{\prime}\right) x$ доминирует вектор $\left(C+C^{\prime}\right) x^{0}$. Полученное противоречие доказывает лемму 4. 
Далее положим

$$
\lambda\left(x^{0}\right)= \begin{cases}\min \left\{\alpha\left(x^{0}, x\right): x \in X \backslash\left\{x^{0}\right\}\right\}, & \text { если } X \neq\left\{x^{0}\right\} \\ \infty, & \text { если } X=\left\{x^{0}\right\}\end{cases}
$$

Через $\lambda^{\prime}\left(x^{0}\right)$ обозначим предельное возмущение коэффициентов векторной функции, при котором задача (2)-(3) имеет в качестве паретовского оптимума точку $x^{0}$.

Поскольку для любой точки $x \in X \backslash x^{0}$ предельное возмущение матрицы $C$, при котором вектор $\left(C+C^{\prime}\right) x$ не доминирует вектор $\left(C+C^{\prime}\right) x^{0}$, задается в силу лемм 3 и 4 значением функции $\alpha\left(x^{0}, x\right)$, верна следующая лемма.

Лемма 5. Справедливо равенство $\lambda\left(x^{0}\right)=\lambda^{\prime}\left(x^{0}\right)$.

Введем обозначение

$$
t\left(x^{0}\right)= \begin{cases}\min _{x \in E^{n} \backslash X} \max \left\{\alpha\left(x^{0}, x\right), \beta(x)\right\}, & \text { если } X \neq E^{n} \\ \infty, & \text { если } X=E^{n}\end{cases}
$$

Лемма 6. Справедливо неравенство $t\left(x^{0}\right) \geqslant \rho_{0}$.

Доказательство. При $\rho_{0}=0$ лемма очевидна. Поэтому будем считать, что $\rho_{0}>0$.

Если $E^{n} \backslash X=\varnothing$, то утверждение леммы очевидно.

Пусть $E^{n} \backslash X \neq \varnothing$. Предположим, что $t\left(x^{0}\right)<\rho_{0}$. Тогда существует такая точка $x \in E^{n} \backslash X$, что $\alpha\left(x^{0}, x\right)<\rho_{0}$ и $\beta(x)<\rho_{0}$. Поэтому согласно лемме 4 точка $x^{0}$ не является $\rho_{0}$-устойчивой относительно точки $x$. Следовательно, с учетом определения 3 существует такая матрица $C^{\prime} \in \mathbf{R}^{k \times n}$, что

$$
\left\|C^{\prime}\right\|<\rho_{0}
$$

а вектор $\left(C+C^{\prime}\right) x$ доминирует вектор $\left(C+C^{\prime}\right) x^{0}$.

Далее, так как $\beta(x)<\rho_{0}$, в силу леммы 2 справедливо неравенство $\beta^{\prime}(x)<\rho_{0}$. Отсюда, по определению числа $\beta^{\prime}(x)$ существуют такие матрица $A^{\prime} \in \mathbf{R}^{m \times n}$ и вектор $b^{\prime} \in \mathbf{R}^{m}$, что расстояние между системами (2) и (4) будет меньше, чем $\rho_{0}$, а $x-$ решение системы (4) при указанных $A^{\prime}$ и $b^{\prime}$.

Собирая все доказанное, убеждаемся, что при выбранных $C^{\prime}, A^{\prime}$ и $b^{\prime}$ расстояние между задачами (1)-(2) и (3)-(4) меньше, чем $\rho_{0}$, но точка $x^{0}$ не является паретовским оптимумом задачи (3)-(4). Полученное противоречие доказывает лемму 6 .

\section{3. Основной результат}

Теорема 1. Для радиуса устойчивости паретовского оптимума $x^{0} k$-критериалъной $(k \geqslant 1)$ задачи (1)-(2) справедлива формула

$$
\rho_{0}\left(x^{0}, A, b, C\right)=\min \left\{\nu\left(x^{0}\right), \lambda\left(x^{0}\right), t\left(x^{0}\right)\right\} .
$$

Доказательство. Методом от противного, используя лемму 1 , легко доказать, что

$$
\rho_{0} \leqslant \nu\left(x^{0}\right)
$$


Из определения числа $\lambda^{\prime}\left(x^{0}\right)$ и леммы 5 вытекает, что

$$
\rho_{0} \leqslant \lambda^{\prime}\left(x^{0}\right)=\lambda\left(x^{0}\right) .
$$

Поэтому с учетом леммы 6

$$
\rho_{0} \leqslant \min \left\{\nu\left(x^{0}\right), \lambda\left(x^{0}\right), t\left(x^{0}\right)\right\} .
$$

Пусть теперь $t_{0}$ - правая часть последнего неравенства. Покажем, что $\rho_{0} \geqslant t_{0}$. Для этого достаточно доказать, что при любом $\varepsilon<t_{0}$ точка $x^{0}-$ паретовский оптимум всякой возмущенной задачи (3)-(4), находящейся от задачи (1)-(2) на расстоянии меньшем, чем $\varepsilon$. Такую возмущенную задачу будем обозначать через $Y(\varepsilon)$.

Поскольку $t_{0} \leqslant \nu\left(x^{0}\right)$, ввиду леммы 1 точка $x^{0}$ является допустимым решением задачи $Y(\varepsilon)$.

Пусть $x$ - произвольное допустимое решение задачи $Y(\varepsilon)$, отличное от $x^{0}$.

Рассмотрим два случая.

В первом случае $x \in X$. Тогда с учетом очевидных неравенств

$$
\left\|C^{\prime}\right\|<\varepsilon<t_{0} \leqslant \lambda\left(x^{0}\right) \leqslant \alpha\left(x^{0}, x\right)
$$

и леммы 3 заключаем, что точка $x^{0}$ является $\varepsilon$-устойчивой относительно точки $x$, т.е. вектор $\left(C+C^{\prime}\right) x$ не доминирует вектор $\left(C+C^{\prime}\right) x^{0}$.

Во втором случае $x \in E^{n} \backslash X$. Тогда из определения величины $\beta^{\prime}(x)$ и леммы 2 следует, что число $\beta(x)$ есть расстояние от системы (2) до ближайшей из систем $(4)$, имеющих точку $x$ в качестве решения. Поскольку $x-$ решение задачи $Y(\varepsilon)$, справедливо неравенство

$$
\beta(x)<\varepsilon
$$

Тогда очевидно, что

$$
\alpha\left(x^{0}, x\right)>\varepsilon,
$$

так как в противном случае с учетом (5) находим, что

$$
t_{0} \leqslant t\left(x^{0}\right) \leqslant \max \left\{\alpha\left(x^{0}, x\right), \beta(x)\right\} \leqslant \varepsilon,
$$

что противоречит неравенству $\varepsilon<t_{0}$.

Далее, из (6) в силу леммы 3 получаем, что и в этом случае вектор $\left(C+C^{\prime}\right) x$ не доминирует вектор $\left(C+C^{\prime}\right) x^{0}$ при $\left\|C^{\prime}\right\|<\varepsilon$.

Следовательно, точка $x^{0}$ - паретовский оптимум задачи $Y(\varepsilon)$.

Теорема доказана.

Как уже отмечалось в [1], величины $\nu\left(x^{0}\right), \lambda\left(x^{0}\right)$ и $t\left(x^{0}\right)$, фигурирующие в формулировке теоремы, имеют вполне определенный смысл: $\nu\left(x^{0}\right)$ и $\lambda\left(x^{0}\right)$ представляют собой предельные возмущения соответственно матрицы ограничений и коэффициентов целевых функций, а $t\left(x^{0}\right)$ определяет совместное влияние этих двух параметров на устойчивость паретовского оптимума $x^{0}$. Поскольку $\nu\left(x^{0}\right)<\infty$, радиус устойчивости $\rho_{0}$ всегда конечное число.

Следуя [2-4], радиусом квазиустойчивости задачи (1)-(2) назовем инфимум расстояния от этой задачи до задач (3)-(4), множество Парето которых не содержит хотя бы один паретовский оптимум задачи (1)-(2). Обозначив этот радиус через $\rho(A, b, C)$, из теоремы 1 легко выводим следующее утверждение. 
Следствие 1. Для радиуса квазиустойчивости векторной задачи (1)-(2) справедлива формула

$$
\rho(A, b, C)=\min _{x^{0} \in P} \min \left\{\nu\left(x^{0}\right), \lambda\left(x^{0}\right), t\left(x^{0}\right)\right\} .
$$

В случае, когда число критериев $k=1$, векторная задача (1)-(2) превращается в обычную (скалярную) задачу булева программирования, а множество Парето $P$ в множество оптимальных решений. Под устойчивостью оптимального решения $x^{0}$ естественно понимать свойство сохранения оптимальности при мальх возмущениях параметров.

Очевидно, что частным случаем нашей теоремы является следующее утверждение.

Следствие 2. Для радиуса устойчивости оптимального решения $x^{0}$ (не обязательно единственного) скалярной $(k=1)$ задачи

$$
\begin{gathered}
c x \rightarrow \max , \\
A_{i} x \leqslant b_{i}, \quad i \in N_{m}, \quad x \in E^{n}
\end{gathered}
$$

справедлива формула

$$
\rho_{0}\left(x^{0}, A, b, c\right)=\min \left\{\min _{i \in N_{m}} \frac{b_{i}-A_{i} x^{0}}{\left\|x^{0}\right\|^{*}+1}, \min _{x \in X \backslash\left\{x^{0}\right\}} \frac{c\left(x^{0}-x\right)}{\left\|x-x^{0}\right\|^{*}}, t\left(x^{0}\right)\right\},
$$

¿de

$$
t\left(x^{0}\right)=\min _{x \in E^{n} \backslash X} \max \left\{\frac{c\left(x^{0}-x\right)}{\left\|x-x^{0}\right\|^{*}}, \max _{i \in N_{m}} \frac{A_{i} x^{0}-b_{i}}{\left\|x^{0}\right\|^{*}+1}\right\}, \quad \text { ecлu } E^{n} \backslash X \neq \varnothing,
$$

$u t\left(x^{0}\right)=\infty$, если $E^{n} \backslash X=\varnothing$.

Отсюда следует очевидный факт, что радиус устойчивости оптимального решения $x^{0}$ скалярной задачи (7)-(8) может быть больше нуля лишь в случае, когда $x^{0}$ - единственное оптимальное решение задачи (7)-(8).

\section{Список литературы}

1. Леонтьев В.К., Мамутов К.Х., Устойчивость решений в задачах линейного булева программирования. Журнал вычисл. матем. и матем. физихи (1988) 28, №10, 1475-1481.

2. Емеличев В.А., Кравцов М.К., Об устойчивости в траекторных задачах векторной оптимизации. Кибернетика и системнъй анализ (1995) №4, 137-143.

3. Емеличев В.А., Кравцов М.К., Подкопаев Д.П., О квазиустойчивости траекторных задач векторной оптимизации. Матем. заметки (1998) 63, №1, 21-27.

4. Емеличев В.А., Кравцов М.К., Подкопаев Д.П., О радиусе квазиустойчивости многокритериальной траекторной задачи. Докл. АН Беларуси (1996) 40, №1, 9-12.

Статья поступила 17.05.1999. 